Monatsschr Kinderheilkd $2021 \cdot 169$ (Suppl 1): S18-S28

https://doi.org/10.1007/s00112-020-01056-x

Angenommen: 9. Oktober 2020

Online publiziert: 18 . November 2020

(C) Der/die Autor(en) 2020, korrigierte

Publikation 2020

\section{Redaktion}

A. Borkhardt, Düsseldorf

S. Wirth, Wuppertal

Wolfgang Rascher ${ }^{1}$. Thomas Klingebiel ${ }^{2}$ Egbert Herting ${ }^{3}$ - Georg F. Hoffmann ${ }^{4}$. Reinhard Berner ${ }^{5}$. Ingeborg Krägeloh-Mann ${ }^{6}$. Jutta Gärtner ${ }^{7}$. Fred Zepp ${ }^{8}$. Ulrike Schara ${ }^{9}$. Deutsche Gesellschaft für Kinder- und Jugendmedizin e. V. (DGKJ)

'Kinder- und Jugendklinik Erlangen, Friedrich-Alexander-Universität Erlangen-Nürnberg, Erlangen, Deutschland

${ }^{2}$ Klink für Kinder- und Jugendmedizin, Universitätsklinikum Frankfurt, Frankfurt, Deutschland

${ }^{3}$ Klink für Kinder- und Jugendmedizin, Universitätsklinikum Schleswig-Holstein, Lübeck, Deutschland

${ }^{4}$ Zentrum für Kinder- und Jugendmedizin, Universitätsklinikum Heidelberg, Heidelberg, Deutschland

${ }^{5}$ Klinik und Poliklinik für Kinder- und Jugendmedizin des Universitätsklinikums Carl Gustav Carus, Technische Universität Dresden, Dresden, Deutschland

${ }^{6}$ Abt. Neuropädiatrie, Entwicklungsneurologie, Sozialpädiatrie, Universitätsklinik für Kinder- und Jugendmedizin, Tübingen, Deutschland

${ }^{7}$ Klink für Kinder- und Jugendmedizin, Universitätsmedizin Göttingen, Göttingen, Deutschland

${ }^{8}$ Zentrum für Kinder- und Jugendmedizin, Universitätsmedizin, Johannes-Gutenberg-Universität Mainz, Mainz, Deutschland

${ }^{9}$ Abteilung für Neuropädiatrie, Zentrum für neuromuskuläre Erkrankungen des Kindes- und Jugendalters, Universitätsklinikum Essen, Universität Duisburg-Essen, Essen, Deutschland

\title{
Arzneimittel für neuartige Therapien - Perspektiven, Chancen, Herausforderungen
}

\section{Stellungnahme der Deutschen Gesellschaft für Kinder- und Jugendmedizin e.V. (DGKJ)}

Basis und rekombinant hergestellte Arzneimittel.

2. Arzneimittel, die Zellen enthalten (somatische Zelltherapie). Die Arzneimittel enthalten entweder substanziell bearbeitete Zellen (z. B. Kultur), oder die Zellen haben im Empfänger nicht dieselbe Funktion wie im Spender (nichthomologer Gebrauch).

3. Tissueengineeringprodukte, biotechnologisch bearbeitete Zellen, Gewebe oder Gewebsprodukte, die eingesetzt werden, um menschliches Gewebe $\mathrm{zu}$ regenerieren, reparieren oder $\mathrm{zu}$ ersetzen.

\section{Beschreibung des therapeu- tischen Potenzials}

Vor allem ATMP der somatischen Gentherapie und Zelltherapie besitzen ein hohes therapeutisches Potenzial für Krankheiten, die sehr früh im Leben beginnen und die bisher nicht behandelbar waren; aber auch für Krankheiten, bei denen die aktuelle Therapie nicht ausreichend wirksam ist oder sich Resistenzen der etablierten Therapie ausbilden.

Der biotechnologische Fortschritt der letzten Jahrzehnte hat nach der molekularen Charakterisierung vieler seltener Krankheiten $\mathrm{zu}$ mutationsspezifischen Therapien geführt (Beispiel zystische Fibrose) [24], und die rekombinanten Technologien haben die Enzymersatztherapie und die Therapie mit monoklonalen Antikörpern ermöglicht. Auch gelang die erste pränatale Therapie zur Korrektur eines Gendefektes durch Proteintransfer über das Fruchtwasser ins Blut des Kindes [25].

Durch den wissenschaftlichen Fortschritt werden nun zunehmend Therapien mit neuartigen Arzneimitteln mittels Gentransfer und genmodifizierten Zellen zur Verfügung stehen, die eine kausale Therapie eines Gendefektes erlauben. Bei angeborenen genetischen Krankheiten, die sich wegen der starken Ausprägung 
Tab. 1 Auswahl von Arzneimitteln für neuartige Therapien (ATMP) für Kinder und Jugendliche. (Aus [31])

\begin{tabular}{|c|c|c|c|c|c|}
\hline Wirkstoff & Arzneimittel & Firma & Zulassung & Indikation & $\begin{array}{l}\text { Klassifizierung } \\
\text { ATMP }\end{array}$ \\
\hline $\begin{array}{l}\text { Onasemnogen abe- } \\
\text { parvovec }\end{array}$ & Zolgensma $^{\circledR}$ & Avexis & 18.05 .2020 & Spinale Muskelatrophie Typ 1 & Gentherapie \\
\hline $\begin{array}{l}\text { Betibeglogen auto- } \\
\text { temcel }\end{array}$ & Zynteglo ${ }^{\circledR}$ & Bluebird bio & 29.05.2019 & Beta-Thalassämie & Gentherapie \\
\hline Voretigen neparvovec & Luxturna ${ }^{\circledR}$ & Novartis & 22.11 .2018 & Retinitis pigmentosa und Leberamaurose & Gentherapie \\
\hline Axicabtagen ciloleucel & Yescarta ${ }^{\circledR}$ & Gilead & 23.08 .2018 & B-Zell-Lymphom (DLBCL und PMBCL) & $\begin{array}{l}\text { Gentherapie } \\
\text { (CAR-T) }\end{array}$ \\
\hline Tisagenlecleucel & Kymriah $^{\circledR}$ & Novartis & 23.08 .2018 & $\begin{array}{l}\text { Akute lymphatische Leukämie und } \\
\text { B-Zell-Lymphom (DLBCL) }\end{array}$ & $\begin{array}{l}\text { Gentherapie } \\
\text { (CAR-T) }\end{array}$ \\
\hline Autologe Zellen & Strimvelis ${ }^{\circledR}$ & Orchard Therapeutics & 26.05.2016 & ADA-SCID & Gentherapie \\
\hline
\end{tabular}

des Gendefektes früh im Leben, d.h. im Säuglings- und im Kleinkindalter, manifestieren, wird eine frühe Diagnosestellung im Hinblick auf neuartige Therapien eine Herausforderung, aber auch eine Domäne der Kinder- und Jugendmedizin werden.

Der theoretische Zusatznutzen der Arzneimittel für neuartige Therapien ist sehr hoch anzusetzen, vor allem, wenn die Therapie wirksam ist, bevor die Krankheit irreversible Funktionsstörungen bis hin zu Neurodegeneration und Organversagen verursacht hat. Arzneimittel für neuartige Therapien sind aber häufig nicht in der Lage, eingetretene irreversible Organschäden aufzuheben. So müssen genetische Krankheiten, für die es eine neue Therapieoption gibt, deutlich früher als bisher diagnostiziert werden.

Bei der Zulassung neuartiger Therapien mit ATMP gelten andere Kriterien als bei der Zulassung herkömmlicher, auch innovativer Arzneimittel, bei denen sich die Wirksamkeit und Sicherheit an der bisherigen, zweckmäßigen Vergleichstherapie in randomisierten kontrollierten Studien orientiert $[8,13]$. Werden neuartige Arzneimittel zugelassen, sind sie noch nicht in ausreichender Anzahl angewendet und häufig noch nicht im direkten Vergleich mit der zweckmäBigen Standardtherapie geprüft worden, sodass die notwendigen Erkenntnisse für eine umfassende Bewertung (Wirksamkeit, Sicherheit, aber auch die genaue Indikation) noch nicht vorliegen können. Dieser Umstand erfordert einen höheren Aufwand der Behandlung und der strukturierten Dokumentation der An- wendung und des Verlaufes. Auch wird der Wert eines Arzneimittels für neuartige Therapien erst mit seiner breiteren Anwendung nach der Zulassung klar, ähnlich wie die Sicherheit eines Arzneimittels nicht mit der Zulassung vollumfänglich bekannt ist.

Ansatzweise hat die Pädiatrie die Probleme der ATMP im Zusammenhang mit der Gentherapie für die spinale Muskelatrophie(SMA) z. T. leidvoll erfahren [29] und beabsichtigt ihre Sichtweise nach Diskussion in einer Arbeitsgruppe darzulegen, damit die Arzneimittel für neuartige Therapien indikationsgerecht angewendet werden, ohne die gebotenen Kriterien für eine rationale und sichere Arzneimittelanwendung für einen wachsenden Organismus außer Acht zu lassen, inklusive einer langfristigen Überwachung der Wirksamkeit und Sicherheit. Mit den Arzneimitteln für neuartige Therapien ergeben sich besondere Herausforderungen an die Diagnostik, die Patientenversorgung und an das Gesundheitssystem.

Bisher sind nach Angaben des Verbandes der forschenden pharmazeutischen Industrie (vfa) zehn Arzneimittel für neuartige Therapien von der Europäischen Arzneimittelagentur (EMA) zugelassenen worden (• Tab. 1). Davon betreffen wenigstens sechs überwiegend Krankheiten der Kinder- und Jugendmedizin. Es ist zu erwarten, dass gerade für Kinder in naher Zukunft weitere neuartige Medikamente entwickelt werden.

\section{Pädiatrische Krankheiten - klinische Beispiele}

Der erfolgreiche Einsatz patienteneigener T-Zellen, die außerhalb des Körpers mit chimären Antigenrezeptoren (CAR) versehen werden, besitzt ein großes Potenzial und erhöht die Therapiechancen von Kindern und Jugendlichen mit akuter B-Zell-Leukämie (CAR-T-Zelltherapie) [6].

Wegen der hohen Erfolgsrate der Gentherapie bei der schwerwiegenden Krankheit spinale Muskelatrophie, die bis vor drei Jahren nicht behandelbar war, wurde auch aufgrund der hohen Wirkstärke von Onasemnogene abeparvovec $\left(\right.$ Zolgensma $\left.{ }^{\circledR}\right)$ die Zulassung in den USA mit den Ergebnissen einer Phase-1Studie mit lediglich 15 Patientinnen und Patienten mit historischen Kontrollen erteilt [1, 20]. Auf Basis der bisherigen Evidenz können keine validen Aussagen zum Risiko-Nutzen-Verhältnis getroffen werden; insbesondere zum möglichen Zusatznutzen im Vergleich zu der gerade erst etablierten Therapie mit dem Antisense-Oligonukleotid Nusinersen $\left(\right.$ Spinraza $\left.{ }^{\circledR}\right)$.

Die Zulassung erfolgte kürzlich auch in Europa durch die Europäische Arzneimittelagentur (EMA), da das Arzneimittel bei Säuglingen mit spinaler Muskelatrophie die Notwendigkeit zur künstlichen Beatmung eindrucksvoll verringern konnte $[10,15]$. Nach 14 Monaten lebten noch 20 von 22 Kindern, die Zolgensma ${ }^{\circledR}$ erhielten, und sie atmeten spontan, während nur ein Viertel unbehandelter Patienten ohne eine Dauerbeatmung überleben würde. Die Studie 
Monatsschr Kinderheilkd 2021 - 169 (Suppl 1):S18-S28 https://doi.org/10.1007/s00112-020-01056-X

(c) Der/die Autor(en) 2020

W. Rascher · T. Klingebiel · E. Herting · G. F. Hoffmann · R. Berner · I. Krägeloh-Mann · J. Gärtner · F. Zepp · U. Schara · Deutsche Gesellschaft für Kinder- und Jugendmedizin e.V. (DGKJ)

\section{Arzneimittel für neuartige Therapien - Perspektiven, Chancen, Herausforderungen. Stellungnahme der Deutschen Gesellschaft für Kinder- und Jugendmedizin e. V. (DGKJ)}

\section{Zusammenfassung}

Arzneimittel für neuartige Therapien (ATMP) wie somatische Gentherapie und Zelltherapie besitzen ein hohes therapeutisches Potenzial für Krankheiten, die sehr früh im Leben beginnen, und die bisher nicht behandelbar waren. Sie werden oft in einem sehr frühen Entwicklungsstadium zugelassen, wenn an wenigen Betroffenen die Wirksamkeit gezeigt wurde und sich ein bisher nie dagewesener Therapieerfolg auftut, vor allem, wenn die Therapie vor Eintritt von Organschäden greift. Dadurch ergeben sich für Pädiater neue arzneimittelrechtliche und ethische Fragen. Um die neuen Behandlungsmöglichkeiten adäquat einzusetzen, muss die Diagnose früher als bisher gestellt werden, oder neue Screeningmethoden müssen zur Verfügung stehen. Denkbar ist, dass das Neugeborenenscreening in zeitkritische Krankheiten in den ersten $72 \mathrm{~h}$ nach Geburt und ein genetisches Screening ( $z$. B. in der 4. bis 5. Lebenswoche) aufgeteilt wird. ATMP sind bei ihrer Zulassung noch nicht in ausreichender Anzahl angewendet worden, sodass die notwendigen Erkenntnisse für Wirksamkeit und Sicherheit noch nicht vorliegen (Nutzen-Risiko-Verhältnis). Deswegen werden sie unter strengen Auflagen in spezialisierten Behandlungszentren nach Qualitätskriterien eingesetzt, die der Gemeinsame Bundesausschuss (G-BA) nach Beratung mit den Fachgesellschaften festlegt. Der Aufwand der Therapie und der Dokumentation des Verlaufes in Registern ist erheblich und muss entsprechend vergütet werden.

Der Wert eines ATMP wird erst mit seiner breiteren Anwendung nach der Zulassung klar, ähnlich wie die Sicherheit eines Arzneimittels nicht mit der Zulassung vollumfänglich bekannt ist. Für die Pädiatrie ergeben sich neue Herausforderungen und Chancen.

\section{Schlüsselwörter}

Gentherapie · Zelltherapie · Neugeborenenscreening · Arzneimittelsicherheit . Ethik

\section{Advanced therapy medicinal products-Perspectives, opportunities and challenges. Statement of the German Society of Pediatrics and Adolescent Medicine (DGKJ)}

Abstract

Advanced therapy medicinal products (ATMP), such as somatic gene therapy and cell therapy, have a high therapeutic potential for diseases that begin very early in life and that were previously untreatable. They are often licensed at a very early stage of development when the efficacy has been shown in a few affected patients and a previously unprecedented therapeutic success is revealed, especially if the therapy is effective before organ damage occurs. This gives rise to new pharmaceutical legal issues and ethical questions for pediatricians.

In order to adequately use the new treatment options the diagnosis must be made earlier than before or new screening methods must be available. It is conceivable that the newborn screening will be divided into timecritical diseases in the first $72 \mathrm{~h}$ after birth and genetic screening, e.g. in the 4 th-5th weeks of life.

ATMP were not used in sufficient numbers when they were licensed, so that the necessary knowledge on efficacy and safety is not yet available (benefit-risk ratio). That is why they are used under strict conditions in specialized treatment centers according to quality criteria that the Federal Joint Committee (G-BA) determines after consulting the specialist competent societies. The expenditure of therapy and documentation of the clinical course in registers is considerable and must be reimbursed accordingly.

The value of an ATMP becomes clear when it is used more widely after approval, just like the safety of a drug is not fully known with the approval. New challenges and opportunities arise for pediatrics.

Keywords

Gene therapy · Cell therapy · Newborn screening $\cdot$ Drug safety $\cdot$ Ethics zeigte auch, dass Zolgensma ${ }^{\circledR}$ Säuglingen helfen kann, mindestens 30 s lang ohne Hilfe zu sitzen. Vierzehn von 22 Kindern unter Zolgensma ${ }^{\circledR}$ konnten dies nach 18 Monaten. Dieser Meilenstein wird bei unbehandelten Kindern mit schweren Formen der Krankheit nie erreicht. Das Gentherapeutikum Zolgensma ${ }^{\circledR}$ erhielt in Europa eine bedingte Genehmigung für das Inverkehrbringen („conditional marketing authorization“) und wurde als Orphan-Drug zugelassen. In Deutschland wurden kürzlich
Handlungsempfehlungen für den Einsatz dieses Gentherapeutikums publiziert [30].

Für weitere Krankheiten vor allem bei Kindern sind Gen- und Zelltherapien in der Entwicklung.

\section{Krankheitsdiagnose und -verlauf, frühzeitige Diagnose- stellung}

Das Auftreten von Krankheitssymptomen, die Einordnung der typischen klinischen Symptome zu einer Krankheitsgruppe und der Einsatz einer spezifischen Diagnostik war und ist der Weg zur Diagnosestellung. Lange Jahre stand die molekulare Diagnostik am Ende der Diagnosestellung. Dies hat sich aber in den letzten Jahren dramatisch geändert, insbesondere wenn eine Konstellation spezifischer Krankheitssymptome auf eine Erkrankung hinwies, bei der sich durch die molekulare Diagnostik die traditionelle, invasive Diagnosestellung erübrigte (z.B. Muskelbiopsie bei V.a. 
Muskeldystrophie, Nierenbiospie bei V.a. Nephronophthise). Grundsätzlich ist eine frühe Diagnosestellung, am besten über ein Neugeborenenscreening, für eine rechtzeitige sachgerechte Therapie und damit für eine normale körperliche und soziale Entwicklung entscheidend.

Deswegen wurden und werden neue diagnostische Algorithmen, einschließlich molekularer Diagnostik, entwickelt, wenn sich neue Therapiemöglichkeiten auftun, da der Therapieerfolg immer größer ist, wenn noch keine bzw. geringe bleibende Organschäden durch die Krankheit aufgetreten sind.

Ein Umdenken in der Diagnosestellung hin zur Frühdiagnose in spezialisierten Zentren wird hier zunehmend notwendig sein, solange die Erkrankung nicht per Neugeborenenscreening erfasst werden kann. Das heißt, alle Pädiater müssen vorbereitet und geschult werden, auf spezifische Krankheitskonstellationen in einem vordergründig unspezifisch imponierenden Krankheitskontext zu achten. Ein Kleinkind z.B., das sich nicht zeitgerecht motorisch entwickelt und eine (hypotone) Bewegungsstörung zeigt, darf nicht einfach der Frühförderung zugeführt werden, ohne dass abgewogen wird, ob gezielte Diagnostik notwendig ist. Es muss geschult werden, worauf besonders zu achten ist, z. B. gibt es Hinweise auf eine Muskelschwäche, auf einen Entwicklungsknick, auf ein auffälliges Entwicklungsprofil mit diskrepant guter kognitiver Entwicklung? Die alleinige Frühförderung und das Abwarten des Erfolges einer Entwicklungsförderung verhindern ggf. eine zeitgerechte Diagnostik und verzögern die spezifische Therapie. Zu oft erfolgen die molekulare Diagnostik und die Diagnosestellung einer genetischen Krankheit erst verspätet bei fortschreitenden und manifesten Symptomen. Dadurch wird eine spezifische Therapie zu spät eingeleitet, da schon irreversible Schäden gesetzt sind.

Der Anteil an seltenen Erkrankungen ist im Kindes- und Jugendalter besonders hoch und damit das Spektrum des natürlichen Krankheitsverlaufes oft unzureichend bekannt. Entsprechend fehlen auch oft belastbare Daten zur Phänotyp-Genotyp-Korrelation und der prädiktiven Rolle von Biomarkern, die es- senziell sind, für Fragen des Neugeborenenscreenings und der Frühdiagnostik. Das Spektrum der Ausprägung und das Fortschreiten der Krankheit können nur durch eine routinemäßige Erfassung aller Krankheitsfälle und ihrer Verläufe mit standardisierten Instrumenten (einschließlich Genotypdaten sowie metabolischen und Imaging-Biomarkern) in krankheitsspezifischen Registern zusammentragen werden. Diese Register, die industrieunabhängig sein müssen, sind essenziell, um über den natürlichen Krankheitsverlaufhinaus auch die Wirksamkeit und den Nutzen von Arzneimitteln für eine neuartige Therapie zu erfassen. In einigen Bereichen (z. B. im Bereich der therapeutischen monoklonalen Antikörper) ist dies nicht gelungen; die Therapieregister sind industrieabhängig. Insbesondere für seltene Krankheiten müssen die Daten in Europa bzw. weltweit und nicht nur in Deutschland zusammengetragen werden. Beispiele dafür sind die Bestrebungen im Rahmen der European Reference Networks (ERN) [27].

Als mögliches Modell für die Einführung von Arzneimitteln für neuartige Therapien bieten sich die kooperativen Studien der Gesellschaft für Pädiatrische Onkologie und Hämatologie (GPOH) an [19]. Den Studiengruppen der GPOH ist es gelungen, durch unabhängige, gemeinsame Studien („investigator-initiated“) zur Therapieoptimierung und durch die zentrale Erfassung der Therapieerfolge einen Mehrwert zu generieren, der allen onkologisch erkrankten Kindern zugutekommt.

\section{Früherkennung durch Screening}

Das Neugeborenenscreeningprogramm dient der Früherkennung angeborener Krankheiten, für die bei früher Diagnosestellung effektive Behandlungsmöglichkeiten bestehen. Nach Beginn des Screenings in den 1960er-Jahren mit der Untersuchung auf Phenylketonurie wurden bis zum Jahre 2000 wenige weitere Zielkrankheiten in das Screeningprogramm aufgenommen. Die Aufnahme neuer Untersuchungsmethoden in das bundesweite Screening erfordert neben dem Nachweis eines großen Nutzens für die Betroffenen eine sehr hohe Sensiti- vität - d.h. möglichst alle Betroffenen werden durch die Untersuchung zuverlässig identifiziert - und Spezifität d.h. möglichst viele Gesunde werden als gesund erkannt. Rasante Entwicklungen in der Therapie und parallel dazu den diagnostischen Möglichkeiten resultieren darin, dass aktuell jährlich zusätzliche Zielkrankheiten in das Neugeborenenscreening aufgenommen werden. Im Jahre 2005 wurden vom G-BA zwölf Stoffwechsel- und zwei endokrinologische Krankheiten für das bundesweite Screening festgelegt. Im Jahr 2016 wurde die Mukoviszidose (zystische Fibrose) als 15. Zielkrankheit aufgenommen, 2018 die Tyrosinämie Typ I und 2019 die schweren kombinierten Immundefekte. 2021 werden aller Voraussicht nach die Sichelzellanämie und die spinale Muskelatrophie hinzukommen, mit weiteren Kandidatenkrankheiten am Horizont. Das Neugeborenenscreening wurde so zur erfolgreichsten Maßnahme der Sekundärprävention, und bis heute konnten in Deutschland ca. 15.000 betroffene Menschen vor schwerer Behinderung oder frühem Tod bewahrt werden [16].

Die neuen durch Screeningverfahren diagnostizierten Krankheiten sind alle selten und erhöhen die Anforderungen und Komplexität der Screeningstrukturen ganz wesentlich. Inzwischen ist fast jedes tausendste in Deutschland geborene Kind (1:1000) von einer der aktuellen Screeningerkrankungen betroffen, und das mit steigender Tendenz. Vor allem die „neuen“ Screeningerkrankungen Mukoviszidose und schwere kombinierte Immundefekte erfordern nach einem positiven Screeningbefund eindeutige Zeitabläufe, Folgeuntersuchungen und Therapieentscheidungen, die nur mithilfe von Zentrumstrukturen möglich sind, wie sie weiter unten für die Anwendung der ATMP ausgeführt sind. Der zukünftig notwendige Einschluss weiterer Erkrankungen in das Neugeborenenscreening erfordert noch weiter verbesserte und strukturierte Screeningkonzepte.

Einerseits erlaubt nur ein allgemeines populationsbezogenes Neugeborenenscreening die vollständige und rechtzeitige Erfassung betroffener Kinder. Auch bei Anwendung propagierter breit 
angelegter Indikationsuntersuchungen bleiben ca. 50\% der Kinder unerkannt. Dieses wurde für das adrenogenitale Syndrom ebenso nachgewiesen wie für die klassische Galaktosämie und die Tyrosinämie Typ I, die sich jeweils mit schweren progredienten Leberschäden manifestieren und ohne spezifische Therapien tödlich verlaufen. Bei anderen Erkrankungen, z. B. einer Muskelschwäche bei der SMA, sind die bleibenden Schäden wegen später Diagnosestellung ebenso gravierend, wie die aktuelle Versorgungsrealität zeigt. Andererseits ist die Zustimmung zu einem Neugeborenenscreening auf Erkrankungen, die noch nicht relativ einfach und $100 \%$ effektiv behandelbar sind (z. B. Hypothyreose) und bei denen noch größere Unsicherheiten bzgl. des Langzeitverlaufes und -therapieintensität bestehen (z.B. Mukoviszidose und spinale Muskelatrophie), und die generell primär genetische Untersuchungstechniken erfordern, nicht so unumstritten, wie bei den bisherigen Screeningerkrankungen. Diese werden von über $99 \%$ der Familien positiv und weitgehend unhinterfragt wahrgenommen. Mittelfristig erscheint ein zweistufiges Screeningkonzept sinnvoll, mit der Fortführung des klassischen und besonders zeitkritischen Neugeborenenscreenings in den ersten $72 \mathrm{~h}$ nach Geburt und einer zweiten Untersuchung z.B. bei der Früherkennungsuntersuchung U3 in der 4. bis 5. Lebenswoche für ein genetisches Screening auf eine große und flexibel erweiterbare Anzahl monogener seltener Erkrankungen nach differenzierter Aufklärung der Familien.

\section{Kriterien für Arzneimittel für neuartige Therapien}

Arzneimittel für neuartige Therapien nutzen den molekularen Defekt oder die molekulare Präsentation krankheitsspezifischer Zellen. Dies erfordert eine frühzeitige klinische und molekulare Diagnose und das Verständnis der Pathophysiologie, idealerweise bevor die Krankheit irreversible Organschäden verursacht hat. Die Arzneimittel für neuartige Therapien werden in einem sehr frühen Entwicklungsstadium der Medikamentenentwicklung zugelassen, die den Ergebnissen einer Phase-1-Studie entsprechen, oft an nur wenigen Patienten und ohne direkte Kontrollgruppe durchgeführt. Allerdings ist die potenzielle Wirkstärke der Therapie groß. Am Beispiel der Gentherapie der spinalen Muskelatrophie Typ 1 und 2 mit Onasemnogene abeparvovec (Zolgensma ${ }^{\circledR}$ ) wird das hohe Therapiepotenzial deutlich, aber auch die Limitationen und ethischen und ökonomischen Herausforderungen im Zulassungs- und im Markteinführungsprozess.

Ganz aktuell entwickelte ein Patient drei Tage nach Applikation der Gentherapie für die spinale Muskelatrophie eine tödlich verlaufende Myokarditis. Ein Zusammenhang mit der Therapie konnte nicht eindeutig widerlegt werden. Zwei weitere Todesfälle bei kindlichen Patienten ereigneten sich in einer Gentherapiestudie für die X-gebundene myotubuläre Myopathie [28].

ATMP erhalten oft vorzeitig eine $\mathrm{Zu}$ lassung, d.h. sie erhalten eine bedingte Genehmigung für das Inverkehrbringen (Conditional marketing authorization). Diese Zulassung wird im Interesse der öffentlichen Gesundheit gewährt, da das Arzneimittel einen bisher nichtgedeckten medizinischen Bedarfbefriedigt, und der Vorteil der sofortigen Verfügbarkeit überwiegt das Risiko des Inverkehrbringens bei einer geringeren Datenlage als üblicherweise erforderlich. Diese Arzneimittel stehen unter erhöhter Beobachtung, d.h., sie unterliegen einem besonderen Meldesystem. Der Nutzen der sofortigen Verfügbarkeit des Arzneimittels überwiegt die Risiken für die öffentliche Gesundheit, die noch genauer untersucht werden müssen. Es ist zu fordern, dass die pharmazeutische Industrie insgesamt oder einzelne Unternehmer regulatorisch dazu verpflichtet werden, im weiteren Verlauf die Kosten einer vollständig unabhängigen Erfassung umfassender Daten zu Wirksamkeit und Sicherheit ihres neuen Medikamentes zu tragen, ohne Einfluss auf die Erhebung und Interpretation der Daten zu nehmen. Die könnte durch „post-authorisation efficacy studies" (PAES) [14] und „post-marketing safety studies“ (PASS) [17] gewährleistet werden.
Alternativ bzw. ergänzend können krankheitsspezifische Register wie in der Kinderonkologie, in die die Behandlungsdaten von über $95 \%$ der Patienten eingehen, helfen, den Stellenwert der neuartigen Therapien zu belegen. Das gelingt, wenn mit der Refinanzierung eine verpflichtende Dokumentation der Wirksamkeit und Sicherheit verankert wird. Im Interesse des Erkenntnisgewinns, aber auch im Interesse der $\mathrm{Pa}$ tienten, sind eine vollumfängliche Meldung schwerwiegender unerwünschter Arzneimittelwirkungen (UAW) auf einem speziellen Meldebogen [23] und eine Meldung bei Wirkungsverlust der Gentherapie zwingend erforderlich.

Die spezifischen und umfangreichen Therapiemaßnahmen sowie die regulatorischen Herausforderungen mit der notwendigen Dokumentation jedes einzelnen Falles setzen eine qualitätsgesicherte Durchführung in spezialisierten Zentren voraus. Dazu sind wenige, aber über die Bundesrepublik ausreichend viele Zentren erforderlich, die auch die Nachbetreuung übernehmen. Hier kann ein Vergleich mit Transplantationszentren erlaubt sein.

In den vergangenen Jahren sind in Deutschland interdisziplinär und multiprofessionell arbeitende Forschungsund Versorgungsstrukturen für Menschen mit seltenen Erkrankungen entstanden, die Zentren für Seltene Erkrankungen: Referenz- oder Typ-A-Zentren und krankheits(gruppen)spezifische Fach- bzw. Typ-B-Zentren [18]. Die TypA-Zentren verfügen über eine Lotsenstruktur zur Lenkung von Patienten an die passenden Ansprechpartner innerhalb des eigenen Zentrums sowie an andere Einrichtungen deutschlandweit, und darüber hinaus koordinieren sie Aufbau, Einsatz und Pflege von Registern und arbeiten mit der Selbsthilfe zusammen. Typ-B-(Fach)-Zentren betreuen Patienten mit einem definierten Krankheitsbild oder einer definierten Gruppe verwandter Erkrankungen und bieten sowohl ambulante wie auch stationäre Versorgung durch interdisziplinäre und multiprofessionelle Teams. Diese Strukturen bieten damit wesentliche Voraussetzungen für die Vorbereitung und Durchführung der Therapien mit 
ATMP sowie die anschließende Langzeitbetreuung der Patienten in besonders qualifizierten Fach-bzw. Typ-B-Zentren.

\section{Abgrenzung zu den existieren- den, anderen Therapieoptionen}

Um die Wirksamkeit von Arzneimitteln für neuartige Therapien in einer breiteren Anwendung zu erfahren, sollte bzw. darf sie nicht mit anderen neuen krankheitsspezifischen Therapien kombiniert werden (z. B. Gentherapie der SMA mit Zolgensma ${ }^{\circledR}$ mit der gleichzeitigen Gabe von Nusinersen, Spinraza ${ }^{\circledR}$ ), sonst kann die Wirksamkeit der Gentherapie nicht beurteilt und unerwünschte Arzneimittelwirkungen können nicht zugeordnet werden. Das gilt natürlich nicht im Notfall, wenn z.B. nach Gentherapie einer Hämophilie eine Blutung bei Mangel an einem Gerinnungsfaktor auftritt. In diesem Fall kann eine alternative krankheitsspezifische Rescue-Therapie lebensrettend sein und muss eingesetzt werden.

Bisher gibt es keine sicheren Kriterien, welche krankheitsspezifische Therapie bei welchem Patienten angezeigt ist, solange keine Daten aus vergleichenden Studien oder zur zweckmäßigen Vergleichstherapie zur Verfügung stehen. Somit fällt die Entscheidung im Einzelfall notwendigerweise durch den behandelnden Arzt nach Diskussion im spezialisierten Behandlungsteam im Zentrum und mit den Sorgeberechtigten.

Auch andere Formen der krankheitsspezifischen Therapie wie Organtransplantation (Leberspende als Enzymersatz) oder allogene Stammzelltransplantation (als Ersatz des funktionellen Proteins) müssen im Vergleich zu Arzneimitteln für neuartige Therapien berücksichtigt werden. Da außer bei einer frühen Diagnose im Screening in der Regel Kinder mit Symptomen Arzneimittel für neuartige Therapien erhalten, wird die bisherige symptomatische Therapie selbstverständlich weitergeführt. Persistieren die Symptome nach der neuartigen Behandlung, wird die symptomatische Therapie, auch mit Heil- und Hilfsmitteln, fortgesetzt und bei Progression der Symptome ausgeweitet.

\section{Indikationen, optimaler Einsatz des Medikaments}

Die sich früh im Kindesalter manifestierenden Gendefekte sind klinisch in der Regel rasch progredient. Jede krankheitsspezifische Therapie und v. a. Arzneimittel für neuartige Therapien müssen deswegen möglichst früh eingesetzt werden, optimal, noch bevor eine Organschädigung eintritt. Dazu muss die Diagnosestellung früh bzw. präsymptomatisch durch Screening erfolgen (s. oben). Bei Nachweis eines Gendefektes muss der Patient umgehend in einem Zentrum vorgestellt werden, in Analogie zur Versorgung von Diagnosen, die mittels Neugeborenenscreening festgestellt werden. Von der Gefährlichkeit der Krankheit hängt es dann ab, ob der Patient notfallmäßig stationär aufgenommen werden muss, oder ob eine Vorstellung z.B. innerhalb von drei Werktagen in der Stoffwechselambulanz bzw. in einem krankheitsspezifischen Fachzentrum (z. B. Immundefektoder Muskelzentrum in einem Zentrum für Seltene Erkrankungen) ausreichend ist.

Ein optimaler Einsatz eines Arzneimittels für neuartige Therapien ist erst möglich, wenn ausreichend Erfahrung mit einer Therapie besteht (eindeutig positives Nutzen-Risiko-Verhältnis, Kenntnis der Differenzialindikation). Dies ist nicht gegeben, wenn neuartige Therapeutika (zu) früh zugelassen werden.

Die Sorgeberechtigen müssen entsprechend aufgeklärt werden, und diese Aufklärung über die angestrebte Wirksamkeit und mögliche unerwünschte Wirkungen, aber auch über alternative Therapien, muss dokumentiert werden. Inwieweit dafür einheitliche Aufklärungsbogen verwendet werden sollen, wird zu diskutieren sein.

Der richtige, rationale, sachgerechte und optimale Einsatz eines Arzneimittels für eine neuartige Therapie kann nur durch Datenerhebung aller Anwendungen der Behandlung im Rahmen von verpflichtenden Studien bzw. Dokumentationen nach der Zulassung gefunden werden.

\section{Qualifikation der Leistungs- erbringer in spezialisierten Zentren}

Sicherlich sind die Anforderungen an die Arzneimitteltherapie für neuartige Therapien anders und auch komplexer als das üblicherweise bei Medikamenten, auch bei neuen innovativen Pharmaka, der Fall ist. Schon bei mutationsspezifischen Pharmaka oder auch einigen Biologika ist die Verordnung an bestimmte Auflagen gebunden, wie z. B. „Die Behandlung sollte von einem Arzt begonnen werden, der in der Diagnose und Behandlung von Erkrankungen erfahren ist." In Anlehnung an diese Festlegung wird gefordert, dass Indikationsstellungen von Arzneimitteln für neuartige Therapie von Ärzten vorgenommen und überwacht werden, die in der Diagnose und Therapie der Krankheiten, die mit neuartigen Therapien behandelt werden, erfahren und in der Lage sind, die besonderen Anforderungen, auch im Hinblick auf die komplexe Behandlung in einem multiprofessionellen Behandlungszentrum für chronisch kranke Kinder, zu leisten. Dies sind in der Regel Kliniken der Hochleistungsmedizin wie Universitätsklinika, die nicht nur krankheitsspezifische Zentren, sondern auch die breite interdisziplinäre Behandlungsoptionen (wie z. B. diverse pädiatrische Subspezialitäten und Kinderintensivmedizin) ausweisen und in ihren Apotheken eine Herstellungserlaubnis vorhalten. Die Schulung des Behandlungsteams, ggf. mithilfe von detailliertem Schulungsmaterial, muss gewährleistet sein. Aktuell erscheinen nur einige Universitätsklinika mit ihren Zentren für Seltene Erkrankungen (ZSE), den Studienzentralen (z. B. der Kinderonkologie und anderen fachspezifischer Studienzentralen) in der Lage, die erhöhten Anforderungen an die Dokumentation zu leisten. Dazu sind geeignete Ressourcen in fachspezifischen Behandlungszentren notwendig.

Auch die Anforderungen an die Nachbetreuung, einschließlich deren Finanzierung, müssen geregelt sein (definierte Anweisungen für obligate Kontrolluntersuchungen). So erfordern die ambulante Nachsorge und Verlaufskontrolle nach Behandlung mit Zolgensma ${ }^{\circledR}$ [30] 
in den ersten 12 Monaten nach Applikation eine engmaschige Anbindung an das spezialisierte Behandlungszentrum zur Durchführung aufwendiger Kontrollen, Aufwände, die wiederum extra vergütet werden müssen.

Die Behandlung mit Arzneimitteln für neuartige Therapien bei Kindern und Jugendlichen ist und wird eine spezielle Aufgabe der Pädiatrie. Die Klinken der Hochleistungsmedizin sind in der Lage, aufgrund ihrer Leistungsfähigkeit diese Aufgabe zu übernehmen. Daraus leitet sich auch ein hohes Entwicklungspotenzial für die Behandlung pädiatrischer Patienten ab. Diese Leistungen müssen allerdings jenseits der DRG-Systematik z. B. über Zentrumszuschläge separat finanziert werden.

Die Qualitätskriterien der Behandlung mit neuartigen Therapien legt der G-BA in Rücksprache mit den Fachgesellschaften fest. Dabei ist darauf zu achten, dass die Meinungen mehrerer Fach- bzw. Konventgesellschaften sowie der Patientenvertretungen Gehör finden.

\section{Arzneimittelrechtliche Aspekte neuartiger Therapien}

Die bisher gültigen arzneimittelrechtlichen Vorgaben müssen eingehalten werden. Der Einsatz neuartiger Therapien muss indikationsgerecht sein und kann nach Abschluss derklinischen Studien bis zur Zulassung nach dem Härtefallprogramm („compassionate use“) erfolgen [3]. Das betreffende neuartige Arzneimittel muss entweder Gegenstand eines Zulassungsantrags oder einer noch nicht abgeschlossenen klinischen Prüfung sein und wird kostenlos für eine Anwendung bei Patienten zur Verfügung gestellt werden, die an einer zu einer schweren Behinderung führenden Erkrankung leiden oder deren Krankheit lebensbedrohend ist und die mit einem zugelassenen Arzneimittel nicht zufriedenstellend behandelt werden können.

Arzneimittel für neuartige Therapien, wie zum Beispiel Zolgensma ${ }^{\circledR}$, werden zu einem sehr frühen Zeitpunkt unter gewissen Vorbehalt zugelassen (Conditional marketing authorization). Dies impliziert, dass im Verlauf weitere Forschungsergebnisse vorgelegt werden müssen. Dies hat auch Konsequenzen für den Compassionate use und zwar dahingehend, dass die Anwendung bis hin zur Zulassung den gleichen Regelungen unterliegen wie der besonderen Überwachung nach der Zulassung. Durch die mögliche Anwendung eines Losverfahrens hat sich eine rechtliche Lücke offenbart, die der Gesetzgeber schließen muss.

Im Rahmen des Härtefallprogramms (Compassionate use) kann das noch nicht zugelassene Medikament einer bestimmten Patientengruppe zur Verfügung gestellt werden. Diese Patientengruppe ist durch die Erkrankung und die Indikation festgelegt und kann nicht durch weitere und vor allem nicht durch willkürlich gewählte Bedingungen eingegrenzt werden. Nach europäischem Recht werden Bedingungen für die Vergabe ("conditions for distribution") im europäischen Arzneimittelrecht den Mitgliedstaaten überlassen [11]. Dabei geht es primär um die Bedingungen der Verschreibung und der Anforderung an die Kompetenz der Anwender (seriöse Verschreibung, z.B. aufgrund von Kriterien der Behandlungsbedürftigkeit oder Erfolgschancen). Nicht durch das europäische oder nationale Recht sind eine Rationierung (mengenmäßige Beschränkung) und die Art der Verteilung des Arzneimittels (einschl. Losverfahren) geregelt [22]. Für Rationierung oder Priorisierung sind staatliche Institutionen aufgrund der gesetzlichen Regelungen zuständig und nicht der pharmazeutische Unternehmer.

Die oben geforderten besonderen Anforderungen an die Dokumentation bei der Anwendung eines bedingt zugelassenen Arzneimittels (Conditional marketing authorization) müssen auch unbedingt auf das Härtefallprogramm übertragen werden, um weitere wissenschaftliche Erkenntnisse zu generieren. In diesem Kontext muss diskutiert werden, ob nicht das Härtefallprogramm, wie jede biomedizinische Forschung, einer ethischen Bewertung durch eine Ethikkommission unterzogen werden muss [5].

Die Europäische Arzneimittelagentur hat rechtliche Rahmenbedingungen für die Anwendung neuartiger Therapien festgelegt, die eingehalten werden müs- sen und in einer Publikation zusammengefasst sind [12]. Darüber hinaus müssen die notwendigen Vorgaben zur biologischen Sicherheit („biosafety level 1“) nach EU-Recht und die Biostoffverordnung berücksichtigt werden.

\section{Ethische Bewertung neuartiger Arzneimittel}

Das Medikament Zolgensma ${ }^{\circledR}$ (Onasemnogene abeparvovec) wurde in den USA vor Kurzem zur Behandlung von Kindern mit spinaler Muskelatrophie Typ 1 als Gentherapeutikum zugelassen. Es verspricht, anders als das bisher zur wiederholten intrathekalen Gabe zugelassene Medikament Spinraza ${ }^{\circledR}$ (Nusinersen), Kinder mit dieser Erkrankung mittels einer einmaligen intravenösen Gabe heilen zu können. Angeboten wird es gegenwärtig zu einem Preis von 1,945 Mio.€. Vor der Zulassung in Deutschland gab es um dieses Medikament heftige Kontroversen. Eltern von Kindern, die an einer spinalen Muskelatrophie erkrankt waren, versuchten, noch vor der Zulassung Krankenkassen zur Finanzierung des Medikaments zu zwingen. Dabei wurden sie unterstützt durch intensive Öffentlichkeitsarbeit verschiedener Medien [4]. Obwohl die gesetzlichen Krankenkassen nicht für die Kosten aufkommen mussten (Urteil des Nordrheinwestfälischen Landessozialgerichts) [26], da für die Therapie der spinalen Muskelatrophie ja bereits ein zugelassenes Medikament zur Verfügung steht, haben mehrere Krankenkassen am Ende dem Druck nachgegeben und die Kosten übernommen. Dieses Verfahren wirft die Frage auf, inwieweit der gesetzliche Regelungsrahmen zur Zulassung von Medikamenten missachtet worden ist. Es muss auch die Frage diskutiert werden, ob Ärzte zum Einsatz von Medikamenten außerhalb der Zulassung und einer fachärztlichen Indikationsstellung durch Eltern oder eine interessierte Öffentlichkeit gezwungen werden können.

Eine weitere Kontroverse war mit diesem Medikament verbunden. Der Pharmakonzern Novartis, der das Medikament herstellt und vertreibt, hatte noch vor der Zulassung angekündigt, $100 \mathrm{Be}$ handlungen mit Zolgensma ${ }^{\circledR}$ zu verlosen. 
Auch diese Verlosungsaktion hat zu einer heftigen Kontroverse geführt. Erstens wird der übliche Weg der Arzneimittelzulassung umgangen; Patienten werden einem Medikament ausgesetzt, bevor es zugelassen und damit auch ausreichend auf Sicherheit und Wirksamkeit überprüft worden ist. Überdies wurde mit dieser Lotterie eine Zufallsauswahl für Patienten getroffen, die in den Genuss eines möglicherweise lebensrettenden Medikaments kommen. Eine korrekte Indikationsstellung für eine Behandlung kann nicht per Los erfolgen, sondern muss immer eine ärztliche Entscheidung auf dem Boden von wissenschaftlichen und klinischen Kriterien sein, die eine umfassende Aufklärung der Familien über Chancen und Risiken voraussetzt. Dieses Verfahren widerspricht zudem dem Gleichheitsgrundsatz der Versorgung mit Arzneimitteln. Fraglich ist auch, ob es sich bei dieser Aktion nicht auch um eine verschleierte, aber sehr wirksame Marketingaktion gehandelt hat, die den Druck von Eltern auf die Zulassungsbehörden erhöhen und eventuell auch die Höhe der Therapiekosten festschreiben helfen sollte.

Gleichzeitig entbrannte auch in den Medien eine Diskussion um die Preisgestaltung, wobei die Firmen die relative Seltenheit der jeweiligen Erkrankung und die hohen Entwicklungskosten von innovativen Arzneimitteln, die wie z. B. in dem Fall von Zolgensma ${ }^{\circledR}$ auch durch Übernahmen anderer Unternehmen bedingt waren, in den Vordergrund rückten. Vergessen wurde dabei, dass die initiale Entwicklung des Medikamentes durch Philanthropie und öffentliche Fördergelder die entscheidenden Hürden genommen hatte. Ohne Zweifel müssen ökonomische Anreize für die forschende Pharmaindustrie vorhanden sein, um Entwicklungen vorantreiben zu können. Mit Sicherheit werden aber viele weitere Gentherapeutika folgen, sodass sich auch aus unternehmerischer Kalkulation eine 100prozentige Kostendeckung mit den Erlösen aus dem Verkauf einer Einzelsubstanz für eine sehr seltene Erkrankung als nicht unbedingt zwangsläufig erweist. Vonseiten der Hersteller wurde zudem auch das Argument ins Spiel gebracht, dass eine sehr frühe gentherapeutische Behand- lung zwar zugegebenermaßen sehr teuer sei, aber sich „für die Solidargemeinschaft der Versicherten“ dennoch lohnen könne, da so "geheilte Patienten“ mutmaßlich über Steuern und Abgaben im weiteren Leben zu einer "Amortisation dieser Investitionen“ beitragen würden. Die Firmen argumentieren dabei, dass allein aus diesem Grunde der hohe Preis dieser Medikamente nicht nur durch direkte Produktentwicklungskosten zu rechtfertigen sei, sondern die Pharmaindustrie durch „solche Investitionen der Gesellschaft langfristig Kosten und den einzelnen Betroffenen sehr viel Leid ersparen würde“, was ebenfalls zu honorieren sei. Warum ein „aus der Lebensbilanz errechneter Gewinn“ aber dann ggf. einer Pharmafirma und nicht eher der Solidargemeinschaft der Krankenversicherten zustehen sollte, erschließt sich nicht.

Der Deutsche Ethikrat hat sich in einer Stellungnahme aus dem Jahr 2011 mit dem Thema „Nutzen und Kosten im Gesundheitswesen - zur normativen Funktion ihrer Bewertung " auseinandergesetzt [9]. Obwohl diese Stellungnahme einige Jahre zurückliegt, bietet sie doch ausreichend Material, um sich in der gegenwärtigen Kontroverse Orientierung zu verschaffen. In seiner Analyse hat der Ethikrat vorausgesehen, dass es angesichts der knappen Ressourcen und der steigenden Preise für Arzneimittel für neuartige Therapien zu einer Diskussion über die Grenzen „kollektiver Finanzierungsbereitschaft" kommen wird. „Diese Grenzen sind nicht gleichzusetzen mit einer moralisch bedenklichen Einschränkung gesellschaftlicher Solidarität“ [9]. Der Ethikrat mahnt, dass vor diesem Hintergrund „Priorisierung, Rationalisierung und Rationierung offen thematisiert werden" sollen. Er lehnt eine „verdeckte Rationierung medizinischer Leistungen“ ab. Vor allem dürfen solche Rationierungsentscheidungen nicht „an den einzelnen Arzt oder die einzelne Pflegekraft delegiert werden“. Entscheidungen über den Umfang solidarisch finanzierter Leistungen sind „ethische Entscheidungen, die im gesellschaftlichen Diskurs und auf politischem Weg getroffen werden müssen“. „Das Prinzip der Menschenwürde und die Grundrechte erfordern einen durch Rechte gesi- cherten Zugang jeden Bürgers zu einer angemessenen Gesundheitsversorgung“. Daher könnten sozioökonomische Formeln, die einen kollektiven Nutzen ermitteln, nicht Grundlage von Verteilungsentscheidungen sein. Ganz entscheidend ist die Forderung des Deutschen Ethikrats, dass angesichts knapper Ressourcen diese nur für Maßnahme eingesetzt werden sollen, die „tatsächlich einen Nutzen erbringen“. Neben der jetzt im Arzneimittelrecht festgelegten „frühen Nutzenbewertung zur Preisfestlegung " muss eine ausführliche Nutzenbewertung unabhängig von Kostenerwägungen, vor allem in Bezug auf die patientenrelevanten Endpunkte (Mortalität, Morbidität, Lebensqualität) durch den G-BA und beratende Institute (z. B. das IQWIG) weiterhin jederzeit möglich sein [9].

Damit greift der Ethikrat eine Forderung auf, die viele wissenschaftliche Verbände und Organisationen seit Langem erheben. Das Zulassungsverfahren, inklusive der Preisgestaltung, ist in den einschlägigen Gesetzen mittlerweile transparent geregelt. Nicht geregelt ist, welche Verfahren eingesetzt werden, um den Einsatz eines bereits zugelassenen Medikaments zu optimieren. In der pädiatrischen Onkologie wurde große Erfahrung gewonnen mit „Therapieoptimierungsstudien“, die den Einsatz seit Langem zugelassener und oft nicht mehr patentgeschützter Medikamente kontinuierlich verbessert haben. Damit sind mit relativ geringen Mitteln erhebliche Therapiefortschritte erzielt worden [19, 21]. Mittlerweile unterliegen diese Therapieoptimierungsstudien dem Arzneimittelrecht und werden wie Arzneimittelstudien behandelt. Damit sind aber auch zwangsläufig die Kosten erheblich gestiegen, und die Finanzierung solcher Studien stellt inzwischen ein großes Problem dar. Die überwiegend privaten Förderer solcher Studien (Deutsche Krebshilfe, Deutsche Kinderkrebsstiftung) und auch die öffentlichen Förderer (BMBF und DFG) haben nur sehr begrenzte Mittel zur Verfügung, um diese teuren Arzneimittelstudien zu finanzieren. Damit ergibt sich das Dilemma, dass die durch ihre Mitgliedsbeiträge finanzierten Krankenkassen erhebliche Mittel aufwenden, für Medikamente, deren Platz in der Therapie 
noch nicht genau bekannt ist und durch weitere Studien noch optimierbar wäre.

Ein guter Ansatz ist der Beschluss des G-BA zur Bewertung des Nutzens über eine Aufnahme von Tisagenlecleucel in die Arzneimittelrichtlinie nach $₫ 35 \mathrm{a}$ SGB V als neues Therapeutikum im Rahmen der Behandlung der akuten lymphatischen B-Zell-Leukämie [2]. In diesem Beschluss werden Anforderungen an die qualitätsgesicherte Anwendung von Tisagenlecleucel in qualifizierten Behandlungseinrichtungen formuliert und die Vergütung geregelt. Die Behandlungseinrichtungen müssen umfangreiche Erfahrungen mit der Behandlung der jeweiligen Erkrankung (u.a. in der Zelltherapie) vorweisen sowie besondere personelle (sowohl im ärztlichen als auch im Pflegedienst) und fachliche Anforderungen erfüllen. $\mathrm{Zu}$ den Anforderungen an Infrastruktur und Organisation gehören beispielsweise die Einrichtung eines Tumorboards, die schriftliche Dokumentation der Teilnehmer und der Beratungsergebnisse, die Einbindung der zuständigen Apotheke durch verbindliche Regelungen, die Nachbarschaft der Behandlungsräume zu einer Intensivstation, SOP für die klinische, apparative und laborchemische Überwachung von Komplikationen und eine ärztliche Betreuung nach Facharztstandard in allen erforderlichen Disziplinen, die rund um die Uhr zur Verfügung stehen muss. Besonders wichtig ist, dass die umfängliche Dokumentation der Patientenschicksale (u.a. Vortherapien, unerwünschte Arzneimittelwirkungen, Art und Ort des Ansprechens, Folgetherapien und das Langzeitüberleben) zur Zulassungsvoraussetzung gehört. Hier müsste allerdings auch klar geregelt werden, wer in welcher Weise die zusätzlichen zur Dokumentation erforderlichen Ressourcen bereitstellt und finanziert, und wie genau eine Kontrolle erfolgen soll.

$\mathrm{Zu}$ einem ähnlichen Schluss ist der Deutsche Ethikrat schon vor 9 Jahren gekommen. Er fordert, dass „Transfer und Versorgungsforschung auszubauen" sind, ebenso wie die vom „Hersteller unabhängige Förderung versorgungsnaher klinischer Studien nach Zulassung eines Medikamentes oder Medizinproduktes". In diesem Zusammenhang ist auch die Forderung nach einer „Publikationspflicht für alle Studien zu sehen, die unabhängig von ihrem Ergebnis erfolgen sollte" [9].

"Schnellschüsse“ wie die unter dem medialen Druck entstandene Verlosungsaktion der "2-Mio.-€-Medizin“ müssen in Zukunft verhindert werden und durch ein strukturiertes Vorgehen auch bei Einzel-/Härtefällen ersetzt werden. Hierzu ist ein konzertiertes Vorgehen zwischen Politik (Gesellschaft), den Betroffenen, den Kostenträgern, den Bundesoberbehörden, dem G-BA, der pharmazeutischen Industrie, aber auch den Behandlern notwendig, wobei auch thematisiert werden muss, welches Vorgehen vor einer Zulassung von innovativen Arzneimitteln gewählt werden soll, gerade wenn "Wunderheilungen" schon in einer sehr frühen Phase der Medikamentenentwicklung in der heute digital vernetzten Welt bei den Betroffenen/den Eltern die Runde machen.

\section{Forderung zur Anwendung neuartiger Therapien}

Arzneimittel für neuartige Therapien erfordern für die Anwender ein besonders hohes Maß an klinischen und organisatorischen Fähigkeiten in der Kooperation mit anderen Fachabteilungen und den Behörden, aber auch an Kooperation mit den Sektoren (Klinik und Praxis) sowie zusätzlichen Ressourcen, auch im Hinblick auf externe Dokumentation (Qualitätssicherung, Studienregister). Die Anwendung neuartiger Pharmaka muss in spezialisierten Behandlungszentren und unter strengen Sicherheitsauflagen erfolgen, auch unter Bildung von Behandlungsnetzwerken, und die vom G-BA festgelegten Qualitätskriterien sind einzuhalten.

Der Verlauf der Behandlung und die Erfassung möglicher Nebenwirkungen müssen systematisch, im Langzeitverlauf und im Vergleich zu anderen Therapien, in nationalen oder europäischen, industrieunabhängigen Registern erfasst und wissenschaftlich ausgewertet werden.

Die Arzneimittel für neuartige Therapien können nur dann erfolgreich angewendet werden und zu einem Erkenntnisgewinn führen, wenn in der klinischen Praxis neue Versorgungsstrukturen entstehen wie z.B. ein Zentrum für Genomische Präzisionsmedizin innerhalb der Fachzentren für Seltene Erkrankungen (s. oben). Eingeschlossen werden muss die Verpflichtung, den Krankheitsverlauf über Jahre systematisch zu dokumentieren und alle schwerwiegenden unerwünschten Arzneimittelwirkungen zu melden. Der Aufwand der Behandlung, einschließlich der Qualitätssicherung, Dokumentation, Meldeverpflichtung und Registerbildung, in den dafür spezialisierten Zentren der Kinder- und Jugendmedizin und den kooperierenden Netzwerken und Praxen muss dabei jedoch adäquat vergütet werden.

\section{Korrespondenzadresse}

Prof. Dr. Dr. h.c. Wolfgang Rascher

Kinder- und Jugendklinik Erlangen, FriedrichAlexander-Universität Erlangen-Nürnberg Loschgestr. 15, 91054 Erlangen, Deutschland wolfgang.rascher@uk-erlangen.de

Funding. Open Access funding enabled and organized by Projekt DEAL.

\section{Einhaltung ethischer Richtlinien}

Interessenkonflikt. Interessenkonflikt: W. Rascher hat ein Honorar für ein wissenschaftliches Gutachten vom BfArM (Expertengruppe off-label) und Aufwendungsentschädigung für Beratungen des G-BA (SMA, $M L D, A A D C)$ erhalten. E. Herting übernahm Beraterund/oder Gutachtertätigkeiten für Chiesi. Er ist Mitglied eines wissenschaftlichen Beirats für Dräger. Er übernahm Vortrags-/Schulungstätigkeiten für Dräger, Chiesi, Pfizer, Milupa, AbbVie, Lyomark, Humana und Nestlé. Seine Forschung wurde von Chiesi und dem BMBF unterstützt. Er besitzt ein Patent für LISA bei Chiesi. Er ist Mitglied bei DGKJ, DGPM, GPP, DGPI, DFG, beim wissenschaftlichen Beirat BÄK irreversibler Hirnfunktionsausfall. Als Aspekt, der von Dritten als Einschränkung der Objektivität wahrgenommen werden könnte, nennt er die wissenschaftliche Zusammenarbeit mit 2 Entwicklern des Surfactant-Produktes Curosurf. Außerdem wurde er als Kongresspräsident von Jahrestagungen der DGKJ und der NDGKJ durch Firmen, wie AbbVie, unterstützt. G.F. Hoffmann erhielt ein Honorar für Gutachter- und Beratungstätigkeiten von PTC Therapeutics. Vortrags- und Schulungstätigkeiten übernahm er für Fa. Takeda. Ein Honorar für eine Autorenschaft zahlte die Firma Danone. Seine Forschung wurde finanziert von der Hopp-Stiftung. R. Berner hat eine Forschungsförderung von den Firmen Lilly, Provention Bio, Cubist Pharmaceuticals und Novartis sowie von der Gesellschaft für Pädiatrische Nephrologie, dem Helmholtz Zentrum für Diabetesforschung München, dem Sächsischen Ministerium für Soziales und Verbraucherschutz, dem Innovationsfonds des Gemeinsamen Bundesausschusses, 
dem Bundesministerium für Bildung und Forschung - Medizin-Informatik-Initiative: Collaboration on rare diseases, dem Sächsischen Ministerium für Soziales und Verbraucherschutz und dem Sächsischen Ministerium für Wissenschaft und Kunst erhalten. Er übernahm Vortrags- und Referententätigkeiten für Nestlé Nutrition, Infectopharm, med update $\mathrm{GmbH}$, Deutsche Akademie für Entwicklungsförderung und Gesundheit des Kindes u. Jugendl. e. V., Berufsverband der Kinder- und Jugendärzte, PädiatrieTag Dresden, Dsai - Patientenorganisation für angeborene Immundefekte, Pädiatrisches Forum Bielefeld, Österreichische Gesellschaft für Kinder- u. Jugendmedizin, Klinikum Niederlausitz $\mathrm{GmbH}$, Deutsche Gesellschaft für Infektiologie (via COCS GmbH), DRK-Kliniken Chemnitz und Lichtenstein und Vereinigung Hamburger Kinderärzte e. V. Er war auch bezahlter Berater für Consilium infectiorum, Fa. Infectopharm, Herausgeberund Autorenschaft - Springer Medizin Verlag und Herausgeber- und Autorenschaft - Georg ThiemeVerlag. I. Krägeloh-Mann hat von Shire Reisekosten und Honorar für die Beratung bei der FDA bezüglich der MLD-Enzymersatztherapie erhalten. J. Gärtner hat in den letzten drei Jahren Honorare für Vorträge und Beratung sowie Reisekostenerstattungen der Firmen Bayer, Biogen, Teva und Novartis sowie eine Forschungsförderung der Firma Novartis erhalten. F. Zepp war als Berater-/Gutachter für IMI und Cure Vac tätig. Er war Berater oder Teil eines wissenschaftlichen Beirats für Cure Vac, das Robert Koch-Institut, das Paul-Ehrlich-Institut, das International Vaccine Institute und WHO / FIDEC, die Universität Antwerpen und erhielt hierfür Reisekosten oder eine Aufwandsentschädigung. Er übernahm außerdem Schulungsoder Vortragstätigkeiten für DGPharM, die Deutschen Akademie für Entwicklungsförderung und Gesundheit des Kindes e. V. und die Österreichischen Gesellschaft für Kinderheilkunde. Ebenfalls hielt er Vorträge für die Uniklinik Carl Gustav Carus Dresden, die Universität Leipzig, das Institut für Medizinische Mikrobiologie und Hygiene, die Akademie des Bistums Mainz und IMWI. Die Veranstalter wurden von CHIESI GmbH, Genzyme $\mathrm{GmbH}$, Shire, Actelion, Biogen, Sanofi Pasteur, Pfizer, MSD, Seqirus gesponsert. Auch hielt er Vorträge auf dem Kongress für Kinder- und Jugendmedizin, dem Repetitorium der DGKJ, für den Dachverband für Technologen/-innen und Analytiker/-innen in der Medizin Deutschland e. V. (DVTA) und für 50 Jahre Boehringer Ingelheim Preis. Er erhielt Honorare als Autor von Springer und Thieme Verlag. U. Schara erhielt Zuwendungen für Berater- und Gutachtertätigkeiten für Santhera, PTC Therapeutics, Novartis, Sarepta, Ipsen, Biogen, Dynacure, Alexion, Avexis, Roche, Sanofi. Für diese Firmen arbeitete sie ebenfalls in wissenschaftlichen Beiräten. Vortrags-/Schulungstätigkeiten übernahm sie für Santhera, PTC Therapeutics, Sarepta und Biogen. Ihre Forschungsvorhaben wurden von PTC Therapeutics, Santhera, Roche, Novartis, Sarepta, Biogen, Italfarmaco und Ultragenyx unterstützt. Sie ist Mitglied bei der Gesellschaft für Neuropädiatrie (GNP), der Deutschen Myasthenie Gesellschaft e. V. (DMG), der Deutschen Duchenne Stiftung (aktion benni\&co), Myositis Netz, German Brain Council (GBC), European Neuromuscular Centre (ENMC) und dem Europäischen Netztwerk TREAT-NMD. T. Klingebiel gibt an, dass kein Interessenkonflikt besteht.

Für diesen Beitrag wurden von den Autoren keine Studien an Menschen oder Tieren durchgeführt. Für die aufgeführten Studien gelten die jeweils dort angegebenen ethischen Richtlinien.

Open Access. Dieser Artikel wird unter der Creative Commons Namensnennung 4.0 International Lizenz veröffentlicht, welche die Nutzung, Vervielfältigung, Bearbeitung, Verbreitung und Wiedergabe in jeglichem Medium und Format erlaubt, sofern Sie den/die ursprünglichen Autor(en) und die Quelle ordnungsgemäßnennen, einen Link zur Creative Commons Lizenz beifügen und angeben, ob Änderungen vorgenommen wurden.

Die in diesem Artikel enthaltenen Bilder und sonstiges Drittmaterial unterliegen ebenfalls der genannten Creative Commons Lizenz, sofern sich aus der Abbildungslegende nichts anderes ergibt. Sofern das betreffende Material nicht unter der genannten Creative Commons Lizenz steht und die betreffende Handlung nicht nach gesetzlichen Vorschriften erlaubt ist, ist für die oben aufgeführten Weiterverwendungen des Materials die Einwilligung des jeweiligen Rechteinhabers einzuholen.

Weitere Details zur Lizenz entnehmen Sie bitte der Lizenzinformation auf http://creativecommons.org/ licenses/by/4.0/deed.de.

\section{Literatur}

1. Al-Zaidy SA, Kolb SJ, Lowes L et al (2019) AVXS101 (Onasemnogene abeparvovec) for SMA1: comparative study with a prospective natural history cohort. J Neuromuscul Dis 6:307-317

2. Gemeinsamer Bundesausschuss (2019) Beschluss des GBA über eine Änderung der ArzneimittelRichtlinie (AM-RL): Anlage XII-Beschlüsse über die Nutzenbewertung von Arzneimitteln mit neuen Wirkstoffen nach § 35a SGB V - Tisagenlecleucel (akute lymphatische B-Zell-Leukämie). https:// www.g-ba.de/downloads/39-261-3700/201903-07_AM-RL-XII_Tisagenlecleucel-DLBCL_D375_BAnz.pdf. Zugegriffen: 25. Juli 2020 (BAnz AT 02.04.2019B2)

3. BfArM Härtefallprogramm. https://www.bfarm. de/DE/Arzneimittel/Arzneimittelzulassung/ KlinischePruefung/CompassionateUse/_node. html.Zugegriffen: 25. Juli 2020

4. BILD-Zeitung (2019) Teuerstes Medikament der Welt rettet Michael (1) das Leben. BILDZeitung 29. Sept. 2019. https://www.bild. de/news/inland/news-inland/zolgensma-2-1millionen-medikament-rettet-michael-1-ausludwigsburg-das-leben-65004438.bild.html. Zugegriffen 31. Oktober 2020

5. Borysowski J, Ehni HJ, Górski A (2017) Ethics review in compassionate use. BMCMed 15:136

6. Buchholz CJ, Hartmann J, Schüssler-Lenz M, KellerStanislawski B (2017) Neue Entwicklung in der Onkologie: CAR-T-Zelltherapie. https://www.pei.de/ SharedDocs/Downloads/DE/newsroom/bulletinarzneimittelsicherheit/einzelartikel/2017-car-tzelltherapie.pdf?_blob=publicationFile\&v=2. Zugegriffen: 25. Juli 2020 (Bulletin zur Arzneimittelsicherheit 4,31-35)

7. Bundesärztekammer (2020) Stellungnahme des Wissenschaftlichen Beirats: Präzisionsmedizin: Bewertung unter medizinisch-wissenschaftlichen und ökonomischen Aspekten. Dtsch Arztebl. https://doi.org/10.3238/baek_sn_praezision_ 2020

8. Bundesinstitut für Impfstoffe und biomedizinische Arzneimittel Arzneimittel für neuartige Therapien - Regulatorische Anforderungen und praktische Hinweise. https://www. pei.de/SharedDocs/Downloads/DE/regulation/ beratung/innovationsbuero/broschuere-atmp. pdf? $\quad$ blob=publicationFile $\& v=4$. Zugegriffen: 25. Juli 2020

9. Deutscher Ethikrat (2011) Nutzen und Kosten im Gesundheitswesen - zur Normativen Funktion ihrer Bewertung. Stellungnahme, Printmediendesign, Hamburg, Seite 1-133, https://www.ethikrat. org/fileadmin/Publikationen/Stellungnahmen/ deutsch/DER_StnAllo-Aufl2_Online.pdf. Zugegriffen 31.Oktober 2020

10. EMA (2020) Assessment report Zolgensma EMA/200482/2020. https://www.ema.europa.eu/ en/documents/assessment-report/zolgensmaepar-public-assessment-report_en.pdf. Zugegriffen: 25 . Juli 2020

11. EMA Guideline on Compassionate Use ofr Medicinal Products, Pursuant to Article 83 of Regulation (EC) No 726/2004, Doc. Ref: EMEA/27170/2006. https://www.ema.europa.eu/ en/documents/regulatory-procedural-guideline/ guideline-compassionate-use-medicinalproducts-pursuant-article-83-regulation-ec-no726/2004_en.pdf.Zugegriffen:25.Juli 2020

12. EMA Legal framework: advanced therapies. http:// www.ema.europa.eu/ema/index.jsp?curl=pages/ regulation/general/general_content_000295. jsp\&mid=WCOb01ac058007f4bb. Zugegriffen: 25 . Juli 2020

13. EMA (2007of) Regulation (EC) No $1394 / 2007$ of the European Parliament and of the Council on advanced therapy medicinal products and amending Directive 2001/83/EC and Regulation (EC) No 726/2004. https://ec.europa.eu/health// sites/health/files/files/eudralex/vol-1/reg_2007_ 1394/reg_2007_1394_en.pdf. Zugegriffen: 25. Juli 2020

14. EMA (2016) Scientific guidance on post-authorisation efficacy studies. EMA/PDCO/CAT/CMDh/ PRAC/

CHMP/261500/2015. https://www.ema.europa. eu/en/documents/scientific-guideline/scientificguidance-post-authorisation-efficacy-studiesfirst-version_en.pdf.Zugegriffen: 25. Juli 2020

15. EMA Zolgensma (onasemnogene abeparvovec). An overview of Zolgensma and why it is authorised in the EU. EMA/173982/2020 EMEA/H/C/004750. https://www.ema.europa. eu/en/documents/overview/zolgensma-eparmedicine-overview_en.pdf. Zugegriffen: 25 . Juli 2020

16. Gramer G, Nennstiel-Ratzel U, Hoffmann GF (2018) 50 Jahre Neugeborenenscreening in Deutschland. Bisherige Ergebnisse und zukünftige Herausforderungen. Monatsschr Kinderheilkd 166:987-993

17. HMA, EMA Guideline on good pharmacovigilance practices (GVP)Module VIII-Post-authorisation safety studies (Rev 3), EMA/813938/2011 Rev $3 *$, 9.10.2017. https://www.ema.europa.eu/en/ documents/scientific-guideline/guideline-goodpharmacovigilance-practices-gvp-module-viiipost-authorisation-safety-studies-rev-3_en.pdf. Zugegriffen: 25. Juli 2020

18. Hoffmann GF, Mundlos C, Dötsch J, Hebestreit H (2020) Seltene Erkrankungen in der Pädiatrie von der Diagnostik und Behandlung einzelner Erkrankungen zum Aufbau von Netzwerkstrukturen. Monatsschr Kinderheilkd. https://doi.org/10. 1007/s00112-020-00978-w

19. Klingebiel T, Jürgens $H$, Glauche I et al (2020) Register und Studien in der Gesellschaft für Pädiatrische Onkologie und Hämatologie (GPOH) - Welchen Beitrag leisten sie zum Fortschritt. Klin Padiatr 232:124-135 


\section{Konsensuspapiere}

20. Mendell JR, Al-Zaidy S, Shell R et al (2017) Single-dose gene-replacement therapy for spinal muscular atrophy. N Engl J Med 377:1713-1722

21. Möricke $A$, Zimmermann $M$, Reiter $A$ et al (2010) Long-term results of five consecutive trials in childhood acute lymphoblastic leukemia performed by the ALL-BFM study group from 1981 to 2000. Leukemia 24:265-284

22. Paul-Ehrlich-Institut (2020) Newsroom - Meldungen - Härtefallprogramm für Onasemnogene abeparvovec-xioi zur Behandlung der spinalen Muskelatrophie akzeptiert. https://www.pei. de/DE/newsroom/hp-meldungen/2020/200203onasemnogne-abeparvovex-xioi.html. Zugegriffen:25. Juli 2020

23. Paul Ehrlich Institut Schwerwiegenden unerwünschten Empfängerreaktion im Zusammenhang mit der Anwendung von ATMPs. https://www.pei.de/SharedDocs/Downloads/DE/ arzneimittelsicherheit/atmp-vigilanz/a1a-atmpformular.pdf? _blob=publicationFile \&v=2.Zugegriffen: 25 . Juli 2020

24. Rubenstein RC (2006) Targeted therapy for cystic fibrosis: cystic fibrosis transmembrane conductance regulator mutation-specific pharmacologic strategies. Mol Diagn Ther 10:293-301

25. Schneider $H$, Faschingbauer $F$, SchuepbachMallepell SN et al (2018) Prenatal correction of X-linked hypohidrotic ectodermal dysplasia. NEngl J Med 378:1604-1610

26. Süddeutsche Zeitung (2020) Medizin - Essen: Teures Medikament Zolgensma: Krankenkasse muss nicht zahlen. Süddeutsche Zeitung 13.03.2020. https://www.sueddeutsche.de/ gesundheit/medizin-essen-teures-medikamentzolgensma-krankenkasse-muss-nicht-zahlendpa.urn-newsml-dpa-com-20090101-20031399-321864.Zugegriffen 31. Oktober 2020

27. Taruscio D, Vittozzi L, Choquet R (2015) National registries of rare diseases in Europe: an overview of the current situation and experiences. Public Health Genom 18:20-25

28. Wilson JM, Flotte TR (2020) Moving forward after two deaths in a gene therapy trial of myotubular myopathy. Hum Gene Ther. https://doi.org/10. 1089/hum.2020.182

29. Ziegler A, Müller-Felber W, Hahn A et al (2020) Spinale Muskelatrophie Gentherapie ohneZulassung. Dtsch Arztebl 116(48):A2232-2234

30. Ziegler A, Wilichowski E, Schara E et al (2020) Handlungsempfehlungen zur Gentherapie der spinalen Muskelatrophie mit Onasemnogene Abeparvovec-AVXS-101. Nervenarzt. https://doi. org/10.1007/s00115-020-00919-8

31. vfa. Die forschenden Pharma-Unternehmen: Übersicht über zentralisiert in der EU zugelassene ATMP. https://www.vfa.de/de/arzneimittelforschung/datenbanken-zu-arzneimitteln/atmp. Zugegriffen 31.Oktober 2020 